\title{
Comparative analysis of perioperative outcomes between robot-assisted partial nephrectomy and open partial nephrectomy: a propensity-matched study
}

\author{
Atsuro Sawada, Takashi Kobayashi, Takehiro Takahashi, Jin Kono, Kimihiko Masui, Takuma Sato, Takeshi \\ Sano, Takayuki Goto, Shusuke Akamatsu, Osamu Ogawa \\ Department of Urology, Kyoto University Graduate School of Medicine, Kyoto 606-8507, Japan. \\ Correspondence to: Prof. Osamu Ogawa, Department of Urology, Kyoto University Graduate School of Medicine, 54, Syogoin \\ Kawaharacho, Sakyo-ku, Kyoto 606-8507, Japan. E-mail: ogawao@kuhp.kyoto-u.ac.jp

\begin{abstract}
How to cite this article: Sawada A, Kobayashi T, Takahashi T, Kono J, Masui K, Sato T, Sano T, Goto T, Akamatsu S, Ogawa O. Comparative analysis of perioperative outcomes between robot-assisted partial nephrectomy and open partial nephrectomy: a propensity-matched study. Mini-invasive Surg 2021;5:6. http://dx.doi.org/10.20517/2574-1225.2020.100
\end{abstract}

Received: 12 Oct 2020 First Decision: 10 Dec 2020 Revised: 16 Dec 2020 Accepted: 20 Jan 2021 Published: 3 Feb 2021

Academic Editor: Toshio Takagi Copy Editor: Yue-Yue Zhang Production Editor: Xi-Jun Chen

\begin{abstract}
Aim: Partial nephrectomy is the standard treatment for small renal tumors; however, it remains unclear which surgical approach from among robot-assisted partial nephrectomy (RAPN) and open partial nephrectomy (OPN) is superior. This study aimed to compare perioperative outcomes of RAPN and OPN performed at a single institution after adjusting for preoperative patient and tumor characteristics using propensity score matching (PSM).
\end{abstract}

Methods: In this retrospective cohort study, patients who underwent RAPN or OPN for a renal mass of cT1-2 NO MO between 2005 and 2020 at our institution were recruited. The study outcomes were perioperative outcomes, complications, and pathological and functional outcomes. PSM was used to account for baseline covariates.

Results: Overall, 131 RAPN and 71 OPN cases were extracted; in addition, 58 cases of RAPN and OPN were selected via PSM. RAPN was superior to OPN in terms of estimated blood loss (10 g vs. $160 \mathrm{~g}, P<0.001$ ), ischemia time ( $23 \mathrm{~min}$ vs. $34 \mathrm{~min}, P<0.001$ ), and hospital duration ( 7 days vs. 12 days, $P<0.001$ ). There were no significant differences in the incidence of perioperative complications or in the rate of positive surgical margins (both $P>$ 0.05). With respect to functional outcomes, the rates of preservation of renal function at both 1 day and 3 months postoperatively were higher with RAPN than with OPN ( $85.3 \%$ vs. $69.1 \%$ and $93.3 \%$ vs. $85.6 \%$ respectively, both $P<0.001$ ).

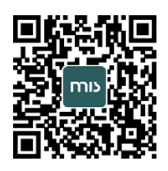


Conclusion: In selected cases, RAPN with warm ischemia appears to preserve renal function equally well or better compared to OPN with cold ischemia.

Keywords: Partial nephrectomy, robot-assisted nephrectomy, open surgery, perioperative outcomes, renal function, propensity score matching

\section{INTRODUCTION}

Partial nephrectomy (PN) for localized renal cell carcinoma has been reported to have oncological outcomes equivalent to those achieved by radical nephrectomy, with preservation of postoperative renal function $^{[1,2]}$. As a result, PN has become the standard treatment for small renal cell carcinomas.

Robot-assisted partial nephrectomy (RAPN) is recognized as a minimally invasive surgical method. Its application as an alternative to open partial nephrectomy (OPN) is rapidly growing ${ }^{[3,4]}$. This is largely due to RAPN's high-definition 3D optical system and flexible wristed instruments that allow surgeons to perform tumor excision and renorrhaphy with an accuracy equal to or greater than that achieved by $\mathrm{OPN}^{[5]}$.

Various studies have compared RAPN and $\mathrm{OPN}^{[6-12]}$. However, because the outcomes of PN are influenced by several factors, including tumor location, anatomical complexity, patient renal function, and operator proficiency, there is some controversy over which surgical approach is superior. Current guidelines do not indicate a preference for one technique over the other, leading to decisions being predominantly made on the basis of the surgeons' expertise and skills ${ }^{[13]}$.

The present study aimed to comprehensively compare the perioperative outcomes of RAPN and OPN performed at a single institution after adjusting for preoperative patient and tumor characteristics using propensity score matching (PSM).

\section{METHODS}

\section{Study population}

This study was approved by the Ethics Committee of Kyoto University Graduate School and Faculty of Medicine (R1581).

We retrospectively collected clinical data of 202 patients with renal masses of cT1-2 cNo cMo diagnosed via CT or MRI who underwent RAPN or OPN between 2005 and 2020 at Kyoto University Hospital. During this period, RAPN was performed by 10 experienced surgeons and OPN was performed by 15 experienced surgeons. The choice of the surgical method (RAPN or OPN) was determined on a case-by-case basis at a preoperative medical conference. However, due to insurance coverage changes that came into effect in 2016, RAPN became the preferred technique. As a general rule, OPN has been applied to patients with a single kidney or chronic kidney disease $(C K D)$ grade 4 or higher $($ eGFR < 30) since 2016. Cases where preoperative imaging was not available were excluded from the study because the anatomic complexity of the tumors could not be accurately determined. Cases with multiple tumors were also excluded for the same reason. Cases in which other surgeries were simultaneously conducted with PN were excluded because perioperative outcomes of PN surgery could not be accurately evaluated.

\section{Surgical technique}

The surgeons at our hospital have received adequate surgical training, have performed many operations at our hospital and other institutions, and are qualified practitioners in Japan. The RAPN procedure employed at our hospital was relatively similar to that reported by Kaouk et al. ${ }^{[14]}$ and was performed using the da 
Vinci S or Xi surgical system (Intuitive, CA, USA). In many cases, the renal artery was clamped using a bulldog clamp. However, when the tumor was superficial and peripheral, the zero ischemia technique was performed, in which the renal artery was not clamped ${ }^{[15]}$. The tumor was then resected along its outline, as confirmed by ultrasonography beforehand. The resection margin was 3-5 $\mathrm{mm}$. If the renal pelvis was open, a central suture was performed to ensure that there was no urine leak before renorrhaphy. The renal artery was declamped after renorrhaphy to check for bleeding from the cut surface.

OPN was performed using the subcostal or flank approach. In most cases, the retroperitoneal approach was used, and OPN under cold ischemia was performed. The renal artery was clamped, and the entire kidney was surrounded by ice slush for 5-10 min before tumor resection ${ }^{[16]}$. Open calyces and bleeding sites were carefully repaired and renorrhaphy was performed. The renal artery was declamped after renorrhaphy.

\section{Outcomes of interest}

The primary and secondary outcomes were examined and compared as evaluation points between RAPN and OPN.

The primary outcomes were perioperative outcomes, namely estimated blood loss (EBL), operative time, ischemia time, and hospital stay. All intraoperative and postoperative complications were also evaluated based on the Clavien-Dindo (CD) classification ${ }^{[17]}$.

The secondary outcomes were pathological and functional outcomes, namely the rates of malignancy, positive surgical margins in malignancy, and pathological stage. Renal function was measured at baseline and at 1 day and 3 months postoperatively based on the estimated glomerular filtration rate (eGFR). The ratio of eGFR at both 1 day and 3 months postoperatively to the baseline eGFR (\% preservation of eGFR) was used as an index to evaluate the postoperative residual renal function.

\section{Covariates}

Patients' preoperative variables were analyzed as covariates, including age at treatment, sex, body mass index (BMI), Charlson comorbidity index $(\mathrm{CCI})^{[18]}$, preoperative eGFR, clinical stage, clinical tumor size (the maximum diameter at preoperative imaging), and tumor side (left or right). Tumor complexity and anatomical characteristics were determined by the urologist and defined using the total "RENAL" nephrometry score ${ }^{[19]}$, namely Radius (tumor size as maximal diameter), Exophytic/endophytic properties of the tumor, Nearness of tumor's deepest portion to the collecting system or sinus, Anterior/posterior descriptor, and the Location relative to the polar line.

\section{Statistical analyses}

Statistical analyses and interpretation of the results were performed according to established guidelines ${ }^{[20]}$. Continuous variables are presented as median and interquartile range (IQR) or mean and standard deviation. Categorical variables are presented as frequency and proportion. Differences in the distribution of continuous and categorical variables between the RAPN and OPN groups were compared using the Mann-Whitney and chi-square tests, respectively.

Adjustments were made using 1:1 nearest-neighbor PSM to account for possible baseline differences between patients who underwent $\mathrm{OPN}$ and $\mathrm{RAPN}^{[21]}$. Propensity scores were calculated using a logistic regression model with odds of receiving RAPN as a dependent variable and age at treatment, sex, BMI, CCI, preoperative eGFR, clinical stage, clinical tumor size, tumor side (right or left), individual RENAL score item, and total RENAL nephrometry score as independent variables. After balanced matching of covariates, the effects of the surgical procedures on outcomes were estimated using the Mann-Whitney and chi-square tests for continuous and categorical variables, respectively. 
All statistical tests were performed using JMP Pro 15.1.0. For all statistical analyses, $P<0.05$ was considered statistically significant.

\section{RESULTS}

\section{Patient characteristics}

As shown in Table 1, a total of 202 patients (131 RAPN and 71 OPN) were included in this study. Prior to PSM, the patients in the cohort who underwent RAPN had a significantly higher BMI $(P=0.006)$ than those who underwent OPN. Furthermore, they had significantly lower RENAL nephrometry scores than those who underwent OPN ( $6.8 \pm 1.61$ vs. $7.5 \pm 1.56$, respectively; $P=0.003)$. A total of 116 cases were compared, comprising 58 RAPN cases and 58 OPN cases that were matched by PSM. In the post-PSM cohort, there were no differences between the RAPN and OPN groups for any of the covariates assessed (all $P>0.05)$ [Figure 1].

\section{Perioperative outcomes and complications}

EBL was significantly higher and hospital stay longer in the OPN group than in the RAPN group [Table 2]. Ischemia time was significantly longer in the OPN group than in the RAPN group; however, cold ischemia time accounted for the majority of the ischemia time in the OPN group.

There were no intraoperative complications in any of the 116 cases selected by PSM. However, postoperative complications occurred in 11 patients who underwent OPN and 8 patients who underwent RAPN.

In both patients who underwent RAPN and OPN, postoperative complications of CD grade 3 or higher included urinomas requiring ureteral stenting and pseudoaneurysms requiring embolization. There was no significant difference in the incidence of postoperative complications between OPN and RAPN [Table 2].

\section{Pathological outcomes}

After PSM, one case of pT2a and one case of pT3a were observed in patients who underwent OPN. There was no difference between the OPN and RAPN groups in terms of positive surgical margins [Table 3].

\section{Functional outcomes}

In the post-PSM cohort, the \% preservation of eGFR at both 1 day and 3 months postoperatively was significantly better in the RAPN group than in the OPN group, although the eGFR at 3 months was not significantly different between the two groups. There were fewer cases with upstaged CKD grades in the RAPN group than in the OPN group (30 cases with OPN vs. 17 cases with RAPN; $P=0.014$ ) [Table 3]. The changes in eGFR for all cases, imperative cases, and elective cases are shown in Figure 2.

\section{Multivariate analysis}

In the pre-PSM cohort, RAPN was found to be a good predictor of EBL $(P<0.0001)$, ischemia time $(P<$ $0.0001)$, transfusion rate $(P=0.019)$, hospital stay $(P<0.0001)$, eGFR $(P<0.0001)$ and \% preservation of eGFR $(P<0.0001)$ at the $3^{\text {rd }}$ postoperative month $(\mathrm{POM})$, and CKD upstaging $(P=0.001)$ via multivariate analysis [Figure 3].

\section{DISCUSSION}

Previous studies have shown mixed results when comparing the outcomes of RAPN and OPN. Simhan et al. ${ }^{[11]}$ compared perioperative outcomes of 281 patients with moderately and highly complex renal lesions. The results showed that RAPN yielded perioperative and functional outcomes similar to OPN, with the additional benefit of shorter hospital stays. Garisto et al. ${ }^{[6]}$ compared perioperative, functional, and 
Table 1. Descriptive characteristics of the study population and tumor characteristics

\begin{tabular}{|c|c|c|c|c|c|c|c|}
\hline \multirow[b]{2}{*}{ Variables } & \multicolumn{3}{|c|}{ Cohort before PSM } & \multirow[b]{2}{*}{ OPN $(n=58)$} & \multicolumn{3}{|c|}{ Cohort after PSM } \\
\hline & OPN $(n=71)$ & RAPN $(n=131)$ & $P$ value & & RAPN $(n=58)$ & $P$ value & SMD \\
\hline Age (yr) & & & 0.065 & & & 0.36 & 0.173 \\
\hline Mean (SD) & $59.4(14.1)$ & $63.0(12.7)$ & & $59.3(13.4)$ & $61.7(14.3)$ & & \\
\hline Sex, $n(\%)$ & & & 0.56 & & & 0.83 & \\
\hline Male & $51(71.8)$ & $99(75.6)$ & & $43(74.1)$ & $44(75.9)$ & & \\
\hline Female & $20(28.2)$ & $32(24.4)$ & & $15(25.9)$ & $14(24.1)$ & & \\
\hline $\mathrm{BMI}, \mathrm{kg} / \mathrm{m}^{2}$ & & & 0.006 & & & 0.78 & 0.052 \\
\hline Mean (SD) & $23.2(4.1)$ & $24.8(3.8)$ & & $23.8(4.2)$ & $23.6(3.5)$ & & \\
\hline $\begin{array}{l}\text { Charlson comorbidity index, } n \\
(\%)\end{array}$ & & & 0.36 & & & 0.90 & \\
\hline 0 & $35(49.3)$ & $76(58.0)$ & & $30(51.7)$ & $29(50.0)$ & & \\
\hline 1 & $11(15.5)$ & $28(21.4)$ & & $11(19.0)$ & $11(19.0)$ & & \\
\hline 2 & $19(26.8)$ & $20(15.3)$ & & $15(25.9)$ & $14(24.1)$ & & \\
\hline$\geq 3$ & $6(8.4)$ & $7(5.3)$ & & $2(3.4)$ & $4(6.9)$ & & \\
\hline eGFR (mL/min/1.73 $\left.\mathrm{m}^{2}\right)$ & & & 0.35 & & & 0.51 & 0.275 \\
\hline Median (IQR) & $64.1(45.1-86.0)$ & $66.3(56.6-77.0)$ & & $64.7(47.1-86.0)$ & $62.1(54.9-73.3)$ & & \\
\hline Imperative case, $n$ (\%) & $34(47.9)$ & $44(33.6)$ & 0.046 & $24(41.3)$ & $25(43.1)$ & 0.85 & \\
\hline Clinical stage, $n(\%)$ & & & 0.19 & & & 1.00 & \\
\hline cT1a & $55(77.5)$ & $111(84.7)$ & & $49(84.5)$ & $49(84.5)$ & & \\
\hline cT1b & $13(18.3)$ & $20(15.3)$ & & $9(15.5)$ & $9(15.5)$ & & \\
\hline cT2a-b & $2(2.8)$ & $0(0)$ & & $0(0)$ & $0(0)$ & & \\
\hline Tumor size $(\mathrm{cm})$ & & & 0.26 & & & 0.63 & 0.089 \\
\hline Median & 3.0 & 2.7 & & 2.5 & 3.0 & & \\
\hline $\mathrm{IQR}$ & $2.1-3.9$ & $2.0-3.5$ & & $1.8-3.6$ & $2.0-3.7$ & & \\
\hline Tumor side, $n(\%)$ & & & 0.45 & & & 1.00 & \\
\hline Left & $37(52.1)$ & $61(46.6)$ & & $28(48.3)$ & $28(48.3)$ & & \\
\hline Right & $34(47.9)$ & $70(53.4)$ & & $30(51.7)$ & $30(51.7)$ & & \\
\hline \multicolumn{8}{|l|}{ RENAL nephrometry score } \\
\hline Radius, $n(\%)$ & & & 0.08 & & & 0.80 & \\
\hline$\leq 4 \mathrm{~cm}$ & $54(76.1)$ & $111(84.7)$ & & $49(84.5)$ & $48(82.8)$ & & \\
\hline $4-7 \mathrm{~cm}$ & $15(21.1)$ & $20(15.3)$ & & $9(15.5)$ & $10(17.2)$ & & \\
\hline$\geq 7 \mathrm{~cm}$ & $2(2.8)$ & $0(0)$ & & $0(0)$ & $0(0)$ & & \\
\hline Exophytic/endophytic & & & 0.17 & & & 0.71 & \\
\hline$\geq 50 \%$ Exophytic & $25(35.2)$ & $53(40.5)$ & & $20(34.5)$ & $23(39.7)$ & & \\
\hline$<50 \%$ Exophytic & $33(46.5)$ & $66(50.4)$ & & $27(46.6)$ & $27(46.4)$ & & \\
\hline Endophytic & $13(18.3)$ & $12(9.1)$ & & $11(19.0)$ & $8(13.8)$ & & \\
\hline $\begin{array}{l}\text { Nearness to the collecting } \\
\text { system }\end{array}$ & & & 0.25 & & & 0.90 & \\
\hline$\geq 7 \mathrm{~mm}$ & $15(21.1)$ & $42(32.1)$ & & $14(24.1)$ & $12(20.7)$ & & \\
\hline 4-7 mm & $16(22.5)$ & $24(19.8)$ & & $11(19.0)$ & $11(19.0)$ & & \\
\hline$\leq 7 \mathrm{~mm}$ & $40(56.3)$ & $65(49.6)$ & & $33(56.9)$ & $35(60.3)$ & & \\
\hline Anterior/posterior, n (\%) & & & 0.52 & & & 0.23 & \\
\hline Anterior & $37(52.1)$ & $60(45.8)$ & & $30(51.2)$ & $28(26)$ & & \\
\hline Posterior & $29(40.9)$ & $56(42.3)$ & & $24(41.4)$ & $31(53.5)$ & & \\
\hline $\begin{array}{l}\text { Location relative to the polar } \\
\text { lines }\end{array}$ & & & 0.02 & & & 1.00 & \\
\hline $\begin{array}{l}\text { Above or below the polar } \\
\text { line }\end{array}$ & $16(22.5)$ & $56(42.8)$ & & $16(27.6)$ & $16(27.6)$ & & \\
\hline $\begin{array}{l}\text { Lesion crosses the polar } \\
\text { line }\end{array}$ & $33(46.5)$ & $44(33.6)$ & & $23(39.7)$ & $23(39.7)$ & & \\
\hline $\begin{array}{l}>50 \% \text { is across the polar } \\
\text { line and crosses the axial } \\
\text { midline entirely between } \\
\text { the polar lines }\end{array}$ & $22(31.0)$ & $31(23.7)$ & & $19(32.8)$ & $19(32.8)$ & & \\
\hline Total score, mean (SD) & $7.5(1.56)$ & $6.8(1.61)$ & 0.003 & $7.4(1.58)$ & $7.4(1.51)$ & 0.95 & 0 \\
\hline
\end{tabular}

RAPN: Robot-assisted partial nephrectomy; OPN: open partial nephrectomy; PSM: propensity score matching; eGFR: estimated glomerular filtration rate; SD: standard deviation; IQR: interquartile range; SMD: standardized mean difference; Imperative case: single kidney, bilateral tumors, or chronic kidney disease (eGFR < 60). 


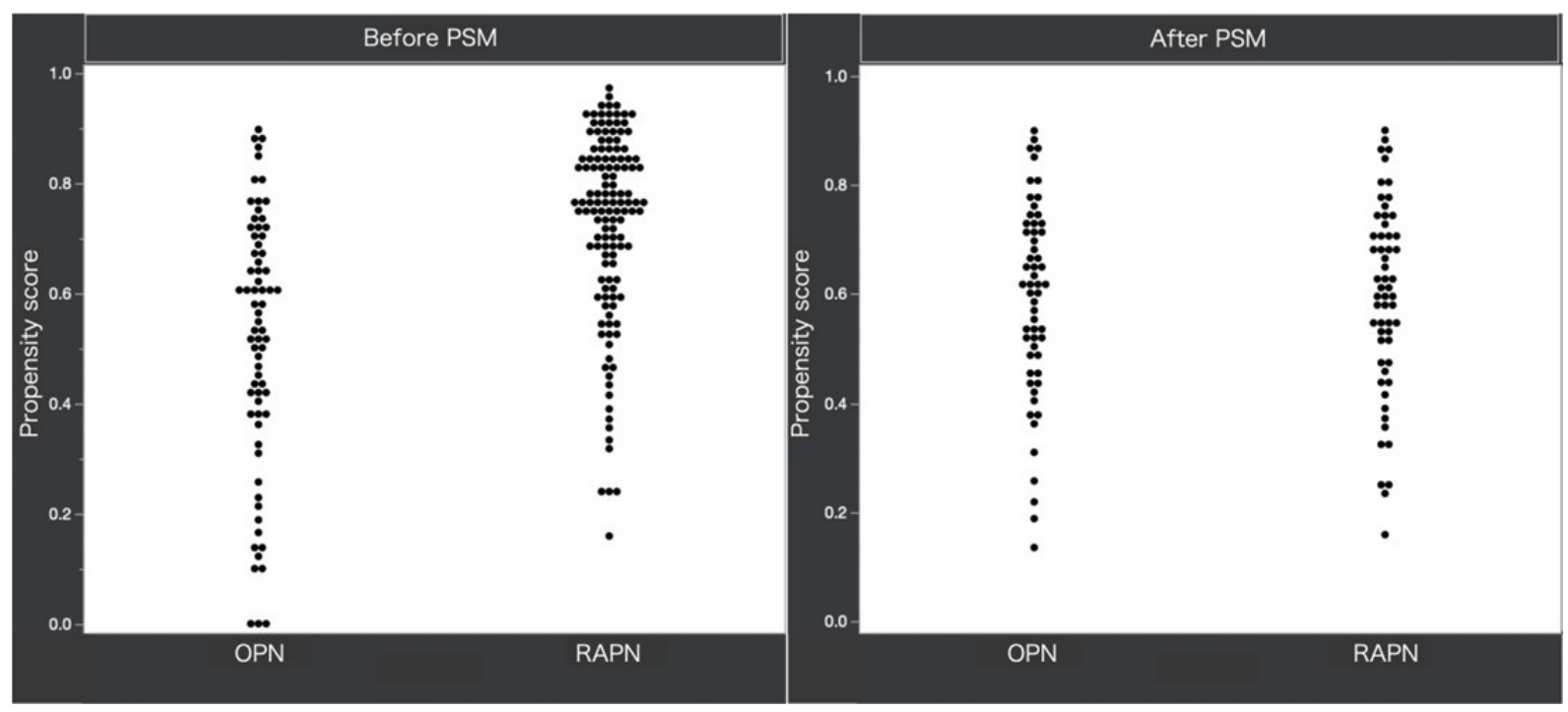

Figure 1. Distribution of the propensity scores. Before PSM (left) and after PSM (right). PSM: propensity score matching; OPN: open partial nephrectomy; RAPN: robot-assisted partial nephrectomy.

Table 2. Comparison of perioperative outcomes between patients treated with RAPN and those treated with OPN after propensity score matching for clinical characteristics

\begin{tabular}{|c|c|c|c|}
\hline Variables & OPN $(n=58)$ & RAPN $(n=58)$ & $P$ value \\
\hline $\mathrm{EBL}(\mathrm{mL})$ & & & $<0.001$ \\
\hline Median (IQR) & $160(90-300)$ & $10(0-60)$ & \\
\hline Operative time ( $\mathrm{min}$ ) & & & 0.003 \\
\hline Median (IQR) & $232(200-260)$ & $258(223-297)$ & \\
\hline Renal artery clamping, $n(\%)$ & & & 0.31 \\
\hline Main artery clamping & $55(94.8)$ & $57(98.3)$ & \\
\hline Zero ischemia & $3(5.2)$ & $1(1.7)$ & \\
\hline Ischemia time (min) & & & $<0.001$ \\
\hline Median (IQR) & $34(26-44)$ & $23(18-28)$ & \\
\hline Cold ischemia time & $27(21-36)$ & 0 & \\
\hline $\begin{array}{l}\text { Transfusion, } n \text { (\%) (including autologous blood } \\
\text { transfusions) }\end{array}$ & $4(6.9)$ & $1(1.7)$ & 0.17 \\
\hline Hospital stay, days & & & $<0.001$ \\
\hline Median (IQR) & $12(9-14)$ & $7(7-9)$ & \\
\hline Conversion to radical nephrectomy, $n(\%)$ & $2(3.5)$ & $2(3.5)$ & 1.0 \\
\hline Overall postoperative complications, $n(\%)$ & $11(19.0)$ & $8(13.8)$ & 0.64 \\
\hline Clavien-Dindo complication $\leq 2$ & $8(13.8)$ & $3(5.2)$ & 0.11 \\
\hline Clavien-Dindo complication $\geq 3$ & $3(5.2)$ & $5(8.6)$ & 0.46 \\
\hline
\end{tabular}

EBL: estimated blood losses, IQR: interquartile range; OPN: open partial nephrectomy; RAPN: robot-assisted partial nephrectomy.

oncological outcomes of RAPN and OPN for the treatment of highly complex renal tumors of 279 cases. Their results indicated that RAPN presents a safe and effective alternative to OPN for highly complex renal tumors, with advantages of reduced blood loss, shorter ischemia time, and shorter length of hospital stay. Other original studies comparing RAPN with OPN have reported that the advantages of RAPN include lower rates of complications ${ }^{[8,22-24]}$. Although, there are many retrospective studies comparing OPN and RAPN, few have compared these surgical approaches in a single-institutional setting using PSM. Because our study analyzed RAPN and OPN from a single institution and matched the patients' backgrounds and tumor complexities using PSM, we believe that our results provide a higher level of evidence. In fact, the use of PSM for all preoperative factors, including the RENAL score, in both groups, which are thought to play important roles in determining the indications and outcomes of RAPN and OPN, resulted in no significant differences between the two groups. 


\begin{tabular}{|c|c|c|c|}
\hline Variables & OPN $(n=58)$ & RAPN $(n=58)$ & $P$ value \\
\hline \multicolumn{4}{|l|}{ Pathological outcomes } \\
\hline Malignancy, $n(\%)$ & $55(94.8)$ & $52(89.7)$ & 0.30 \\
\hline Positive surgical margins, $n(\%)$ & $0 / 55(0)$ & $1 / 52(1.9)$ & 0.50 \\
\hline Stage at final pathology & & & 0.30 \\
\hline pT1a & $50(86.2)$ & $45(77.6)$ & \\
\hline pT1b & $3(5.2)$ & $6(10.3)$ & \\
\hline pT2a-b & $1(1.7)$ & $0(0)$ & \\
\hline рТ3a & $1(1.7)$ & $0(0)$ & \\
\hline uncertain & $3(5.2)$ & $7(12.1)$ & \\
\hline \multicolumn{4}{|l|}{ Functional outcomes } \\
\hline \multicolumn{4}{|l|}{ eGFR at POD $1, \mathrm{~mL} / \mathrm{min} / 1.73 \mathrm{~m}^{2}$} \\
\hline Median (IQR) & $44.4(32.3-64.1)$ & $53.1(40.8-66.6)$ & 0.047 \\
\hline $\begin{array}{l}\% \text { preservation of eGFR at POD } 1 \\
\text { compared with baseline, (\%) }\end{array}$ & $69.1(40.8-66.6)$ & $85.3(72.0-95.4)$ & $<0.001$ \\
\hline \multicolumn{4}{|l|}{ eGFR at $3 r d P O M, m L / m i n / 1.73 \mathrm{~m}^{2}$} \\
\hline Median (IQR) & $56.5(41.9-72.7)$ & $58.3(48.9-72.0)$ & 0.19 \\
\hline $\begin{array}{l}\% \text { preservation of eGFR at 3rd POM } \\
\text { compared with baseline, (\%) }\end{array}$ & $85.6(78.6-88.6)$ & $93.3(83.4-100.9)$ & $<0.001$ \\
\hline CKD upstaging at 3rd POM, $n(\%)$ & $30(51.7)$ & $17(29.3)$ & 0.014 \\
\hline
\end{tabular}

RAPN: Robot-assisted partial nephrectomy; OPN: open partial nephrectomy; eGFR: estimated glomerular filtration rate; IQR: interquartile range; POD: postoperative day; POM: postoperative month; CKD: chronic kidney disease.

The results of our study indicate that RAPN is superior to OPN in terms of EBL, ischemia time, and length of hospital stay. In OPN, cases with long ischemia time of more than 40 min were observed, which were caused by difficulty in suturing and hemostasis owing to difficulty in visualizing the site of the opening of the renal pelvis and bleeding point. We found no significant differences in the incidence of perioperative complications or in the rate of positive surgical margins. With respect to functional outcomes, the rates of preservation of renal function at both 1 day and 3 months postoperatively were higher and the rates of CKD grade upstaging were lower for patients who underwent RAPN than for those who underwent OPN.

There are two possible explanations for the higher rate of preserved renal function in patients who underwent RAPN. One is the difference in the volume of nephron loss during PN. The high-definition 3D optical system and flexible wristed instruments used in RAPN result in lower levels of nephron loss in the resection margin compared with OPN. However, this is merely a predictive interpretation because it is not possible to retrospectively and accurately measure the safety margin in all cases.

Another explanation is the difference in the length of ischemia time. It is known that cold ischemia suppresses damage to the remaining kidney even after $30 \mathrm{~min}^{[25,26]}$. However, although there is clear evidence regarding the protective role of renal cooling in the context of impaired renal function, some studies have suggested that prolonged cold ischemia times and short warm ischemia times also cause nephron damage ${ }^{[27,28]}$. Considering the results of this study, even when cold ischemia using ice slush was performed, it appears that if the ischemia time becomes longer, a shorter period of warm ischemia may be more advantageous for preserving renal function than a longer period of cold ischemia. A previous retrospective study found similar results; the OPN group with cold ischemia had a longer ischemia time, and no significant eGFR advantage was found in favor of OPN. In addition, the trend toward GFR recovery was better in the RAPN group, although it did not reach statistical significance ${ }^{[6]}$.

In studies comparing the effects of cold and warm ischemia and ischemia time on renal function, results showed that when ischemia lasted for $30 \mathrm{~min}$ or longer, renal function was better preserved with cold ischemia. This is because cold ischemia reduces the diffuse and irreversible damage to parenchyma 


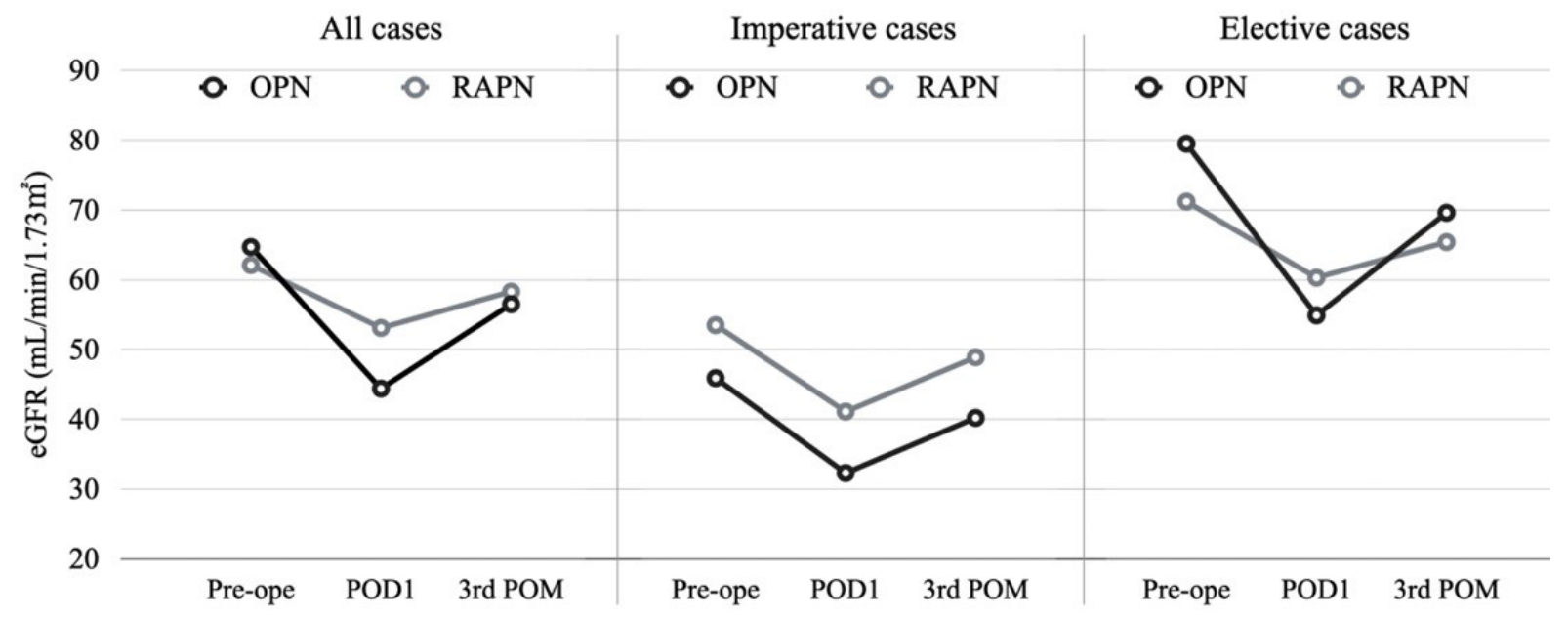

Figure 2. Pre- and post-operative changes in median eGFR in OPN and RAPN for all, imperative, and elective cases. eGFR: Estimated glomerular filtration rate; OPN: open partial nephrectomy; RAPN: robot-assisted partial nephrectomy; Imperative case: single kidney, bilateral tumors, or chronic kidney disease $($ eGFR $<60)$.

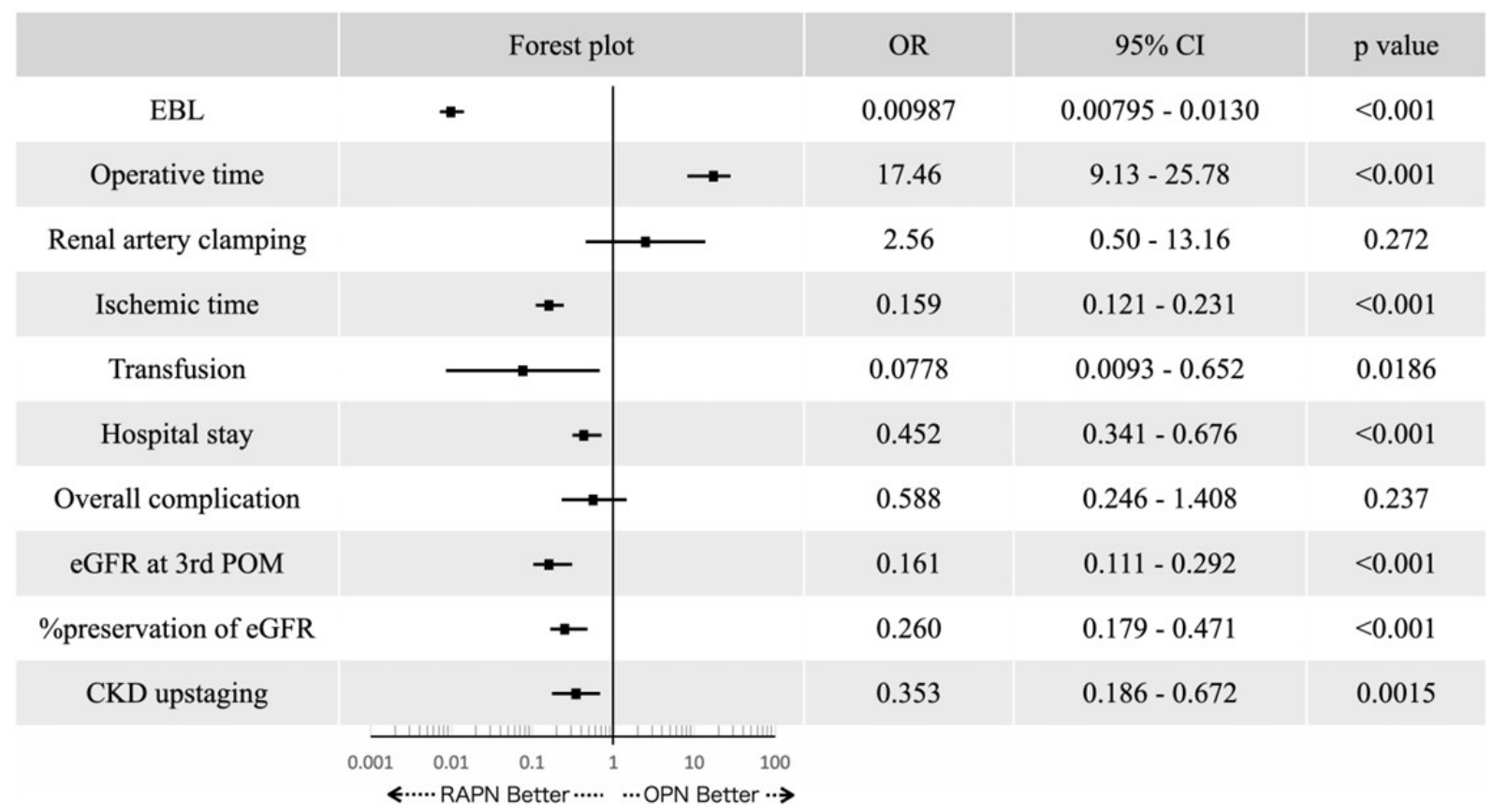

Figure 3. Multivariate logistic regression tests the impact of RAPN vs. OPN on each perioperative outcome according to each OPN and RPN group before matching.

caused by prolonged warm ischemia ${ }^{[29-35]}$. However, when ischemia time was less than 20 min, the preservation of renal function was excellent and no significant difference was observed between cold and warm ischemia ${ }^{[25-28]}$. In light of our results, even in cases where renal function preservation is strongly desired, RAPN with warm ischemia presents a good option if ischemia time is expected to be short. This is evidenced by the equal or greater postoperative renal function achieved with RAPN over OPN. Furthermore, RAPN seems to have some advantages over OPN in terms of other perioperative outcomes. In fact, a study comparing RAPN and OPN for patients with a solitary kidney also concluded that RAPN may offer comparable perioperative and short-term functional outcomes compared with OPN, assuming careful patient selection and adequate surgical experience ${ }^{[36]}$. 
In contrast, cold ischemia should be selected in cases where the tumor is anatomically complex and when the ischemia time is expected to be prolonged. The shorter the cold ischemia period, the better the postoperative renal function is. Considering this, OPN should be prioritized when it can ensure a faster and more accurate resection and renorrhaphy in cases with complex tumors.

In this study, 73 cases in RAPN and 13 cases in OPN were excluded by PSM. Excluded cases included patients in the RAPN group who were relatively older and had a higher BMI and lower RENAL score, and those in the OPN group who were relatively younger and had larger tumor diameters. Therefore, the results of this study may not necessarily apply to such excluded cases.

In recent years, there has been an increasing number of reports confirming that RAPN can be safely used for the resection of complex or large tumors ${ }^{[6,37-39]}$. In this study and other reports, RAPN was shown to have equivalent or better outcomes compared with OPN in many aspects of the perioperative results. This suggests that RAPN is a viable surgical option for the resection of complex and large tumors in the future. However, this hypothesis is based on the premise that the surgeon has sufficient technical proficiency in robotic surgery. Therefore, it is necessary to select an appropriate surgical method according to the surgeon's and the institution's level of proficiency in robotic surgery, taking into consideration the complexity of the tumor and patient factors.

This study had several limitations. First, the sample size of the study was relatively small. Furthermore, it was nonrandomized and retrospective in nature; thus, it was subject to the inherent limitations of a retrospective analysis of observational data, possibly making it difficult to obtain original results. Second, the results of the PSM in this study may be generalized only among those within the propensity score range included in the paired analysis and may not be applicable to those outside this range. Third, different surgeons were involved in this study, which might be seen as a source of biases because different phases of different learning curves were included and might have influenced the results. Fourth, the timing of the surgery (i.e., pre- or post- 2016) was another limitation because more recent cases underwent RAPN and older cases predominantly underwent OPN, as RAPN has been covered by insurance in Japan since 2016 . Finally, this study used data collected from a single center with a high incidence of kidney cancer and cannot be generalized to providers with different characteristics.

In conclusion, this study compares the perioperative outcomes of RAPN and OPN performed at a single institution. Our results indicate that RAPN with warm ischemia preserves renal function equally well or better than does OPN with cold ischemia in selected cases with short ischemic times.

\section{DECLARATIONS}

\section{Acknowledgments}

The authors would like to thank Enago (www.enago.jp) for the English language review.

\section{Authors' contributions}

Made substantial contributions to the conception and design of the study and performed data analysis and interpretation: Sawada A

Performed data acquisition as well as provided administrative, technical, and material support: Takahashi T, Kono J, Masui K, Sato T, Sano T, Goto T

Drafted the article or revised it for critically important intellectual content and approved the final version: Kobayashi T, Akamatsu S, Ogawa O

\section{Availability of data and materials}

Not applicable. 


\section{Financial support and sponsorship}

None.

\section{Conflicts of interest}

All authors declare that there are no conflicts of interest.

\section{Ethical approval and consent to participate}

This study was approved by the Ethics Committee of Kyoto University Graduate School and Faculty of Medicine (reference number: R1581).

\section{Consent for publication}

Not applicable.

\section{Copyright}

(C) The Author(s) 2021.

\section{REFERENCES}

1. MacLennan S, Imamura M, Lapitan MC, et al; UCAN Systematic Review Reference Group. EAU Renal Cancer Guideline Panel. Systematic review of oncological outcomes following surgical management of localised renal cancer. Eur Urol 2012;61:972-93.

2. Van Poppel H, Da Pozzo L, Albrecht W, et al. A prospective, randomised EORTC intergroup phase 3 study comparing the oncologic outcome of elective nephron-sparing surgery and radical nephrectomy for low-stage renal cell carcinoma. Eur Urol 2011;59:543-52.

3. Kaouk JH, Spana G, Hillyer SP, White MA, Haber GP, Goldfarb D. Robotic-assisted laparoscopic partial nephrectomy for a 7-cm mass in a renal allograft. Am J Transplant 2011;11:2242-6.

4. Benway BM, Bhayani SB, Rogers CG, et al. Robot-assisted partial nephrectomy: an international experience. Eur Urol 2010;57:815-20.

5. Maurice MJ, Ramirez D, Kaouk JH. Advances in robotic-assisted treatments for renal cell carcinoma. Curr Opin Urol 2016;26:417-23.

6. Garisto J, Bertolo R, Dagenais J, et al. Robotic versus open partial nephrectomy for highly complex renal masses: Comparison of perioperative, functional, and oncological outcomes. Urol Oncol 2018;36:471.e1-9.

7. Larcher A, Capitanio U, De Naeyer G, et al. Is Robot-assisted Surgery Contraindicated in the Case of Partial Nephrectomy for Complex Tumours or Relevant Comorbidities? A Comparative Analysis of Morbidity, Renal Function, and Oncologic Outcomes. Eur Urol Oncol 2018;1:61-8

8. Ficarra V, Minervini A, Antonelli A, et al. A multicentre matched-pair analysis comparing robot-assisted versus open partial nephrectomy. BJU Int 2014;113:936-41.

9. Mano R, Schulman A, Hakimi AA, et al. Cost comparison of open and robotic partial nephrectomy using a short postoperative pathway. Urology 2015;85:596-603.

10. Han KS, Song GH, You D, et al. Comparison of Hand-Assisted Laparoscopic vs Robot-Assisted Laparoscopic vs Open Partial Nephrectomy in Patients with T1 Renal Masses. J Endourol 2017;31:374-9.

11. Simhan J, Smaldone MC, Tsai KJ, et al. Perioperative outcomes of robotic and open partial nephrectomy for moderately and highly complex renal lesions. J Urol 2012;187:2000-4.

12. Lee S, Oh J, Hong SK, Lee SE, Byun SS. Open versus robot-assisted partial nephrectomy: effect on clinical outcome. J Endourol 2011;25:1181-5

13. Ljungberg B, Albiges L, Abu-Ghanem Y, et al. European Association of Urology Guidelines on Renal Cell Carcinoma: The 2019 Update. Eur Urol 2019;75:799-810.

14. Kaouk JH, Khalifeh A, Hillyer S, Haber GP, Stein RJ, Autorino R. Robot-assisted laparoscopic partial nephrectomy: step-by-step contemporary technique and surgical outcomes at a single high-volume institution. Eur Urol 2012;62:553-61.

15. Gill IS, Eisenberg MS, Aron M, Berger A, Ukimura O, et al. 'Zero Ischemia' partial nephrectomy: novel laparoscopic and robotic technique. Eur Urol 2011;59:128-34.

16. Russo P. Partial nephrectomy for renal cancer (part II): the impact of renal ischaemia, patient preparation, surgical approaches, management of complications and utilization. BJU Int 2010;105:1494-507.

17. Clavien PA, Barkun J, de Oliveira ML, et al. The Clavien-Dindo classification of surgical complications: five-year experience. Ann Surg 2009;250:187-96.

18. Charlson ME, Pompei P, Ales KL, Mackenzie C. A new method of classifying prognostic comorbidity in longitudinal studies: Development and validation. J Chronic Dis 1987;40:373-83.

19. Kutikov A, Uzzo RG. The R.E.N.A.L. nephrometry score: a comprehensive standardized system for quantitating renal tumor size, location and depth. J Urol 2009;182:844-53.

20. Assel M, Sjoberg D, Elders A, et al. Guidelines for reporting of statistics for clinical research in urology. BJU Int 2019;123:401-10.

21. D'Agostino Sr RB. Adjustment methods: propensity score methods for bias reduction in the comparison of a treatment to a nonrandomized control group. In Tutorials in Biostatistics, Chichester, UK: John Wiley \& Sons; 2005. p.67-83. 
22. Minervini A, Vittori G, Antonelli A, et al. Open versus robotic-assisted partial nephrectomy: a multicenter comparison study of perioperative results and complications. World J Urol 2014;32:287-93.

23. Mari A, Antonelli A, Bertolo R, et al. Predictive factors of overall and major postoperative complications after partial nephrectomy: Results from a multicenter prospective study (The RECORd 1 project). Eur J Surg Oncol 2017;43:823-30.

24. Peyronnet B, Seisen T, Oger E, et al; French Comittee of Urologic Oncology (CCAFU). Comparison of 1800 Robotic and Open Partial Nephrectomies for Renal Tumors. Ann Surg Oncol 2016;23:4277-83.

25. Funahashi Y, Yoshino Y, Sassa N, Matsukawa Y, Takai S, Gotoh M. Comparison of warm and cold ischemia on renal function after partial nephrectomy. Urology 2014;84:1408-12.

26. Volpe A, Blute ML, Ficarra V, et al. Renal Ischemia and Function After Partial Nephrectomy: A Collaborative Review of the Literature. Eur Urol 2015;68:61-74.

27. Lane BR, Russo P, Uzzo RG, et al. Comparison of cold and warm ischemia during partial nephrectomy in 660 solitary kidneys reveals predominant role of nonmodifiable factors in determining ultimate renal function. $J$ Urol 2011;185:421-7.

28. Mir MC, Campbell RA, Sharma N, et al. Parenchymal volume preservation and ischemia during partial nephrectomy: functional and volumetric analysis. Urology 2013;82:263-8.

29. Lane BR, Babineau DC, Poggio ED, et al. Factors predicting renal functional outcome after partial nephrectomy. J Urol 2008;180:23638; discussion 2368-9.

30. Thompson RH, Frank I, Lohse CM, et al. The impact of ischemia time during open nephron sparing surgery on solitary kidneys: a multiinstitutional study. J Urol 2007;177:471-6.

31. Thompson RH, Lane BR, Lohse CM, et al. Every minute counts when the renal hilum is clamped during partial nephrectomy. Eur Urol 2010;58:340-5.

32. Porpiglia F, Fiori C, Bertolo R, et al. The effects of warm ischaemia time on renal function after laparoscopic partial nephrectomy in patients with normal contralateral kidney. World J Urol 2012;30:257-63.

33. Funahashi Y, Hattori R, Yamamoto T, Kamihira O, Kato K, Gotoh M. Ischemic renal damage after nephron-sparing surgery in patients with normal contralateral kidney. Eur Urol 2009;55:209-15.

34. Becker F, Van Poppel H, Hakenberg OW, et al. Assessing the impact of ischaemia time during partial nephrectomy. Eur Urol 2009;56:625-34.

35. Patel AR, Eggener SE. Warm ischemia less than 30 minutes is not necessarily safe during partial nephrectomy: every minute matters. Urol Oncol 2011;29:826-8.

36. Zargar H, Bhayani S, Allaf ME, et al. Comparison of perioperative outcomes of robot-assisted partial nephrectomy and open partial nephrectomy in patients with a solitary kidney. $J$ Endourol 2014;28:1224-30.

37. Buffi NM, Saita A, Lughezzani G, et al; ERUS Scientific Working Group. Robot-assisted Partial Nephrectomy for Complex (PADUA Score $\geq 10$ ) Tumors: Techniques and Results from a Multicenter Experience at Four High-volume Centers. Eur Urol 2020;77:95-100.

38. Hennessey DB, Wei G, Moon D, et al. Strategies for success: a multi-institutional study on robot-assisted partial nephrectomy for complex renal lesions. BJU Int 2018;121 Suppl 3:40-7.

39. Beksac AT, Okhawere KE, Elbakry AA, et al. Management of high complexity renal masses in partial nephrectomy: A multicenter analysis. Urol Oncol 2019;37:437-44. 